\title{
Production and Hadronic Decays of Higgs Bosons in Heavy-Ion Collisions
}

\author{
Edmond L. Berger, ${ }^{1, *}$ Jun Gao, ${ }^{2, \dagger}$ Adil Jueid, ${ }^{2, \$}$ and Hao Zhang ${ }^{3,4, \S}$ \\ ${ }^{1}$ High Energy Physics Division, Argonne National Laboratory, Argonne, Illinois 60439, USA \\ ${ }^{2}$ INPAC, Shanghai Key Laboratory for Particle Physics and Cosmology, School of Physics and Astronomy, \\ Shanghai Jiao Tong University, Shanghai 200240, China \\ ${ }^{3}$ Theoretical Physics Division, Institute of High Energy Physics, Chinese Academy of Science, Beijing 100049, China \\ ${ }^{4}$ School of Physics, University of Chinese Academy of Science, Beijing 100049, China
}

(Received 24 April 2018; revised manuscript received 28 June 2018; published 1 February 2019)

\begin{abstract}
We examine Higgs boson production and decay in heavy-ion collisions at the LHC and future colliders. Owing to the long lifetime of the Higgs boson, its hadronic decays may experience little or no screening from the hot and dense quark-gluon plasma, whereas jets from hard scattering processes and from decays of the electroweak gauge bosons and the top quark suffer significant energy loss. This distinction can lead to enhanced signal to background ratios in hadronic decay channels and thus, for example, provide alternative ways to probe the Yukawa coupling of the Higgs boson to the bottom quark and its lifetime.
\end{abstract}

DOI: 10.1103/PhysRevLett.122.041803

Introduction.-The successful operation of the CERN Large Hadron Collider (LHC) led to the discovery of the Higgs boson, the final piece of the standard model (SM) $[1,2]$ of particle physics. Precise measurements of the properties and couplings of the Higgs boson are now required for a refined understanding of the nature of electroweak symmetry breaking and for searches for new physics beyond the SM. This pursuit has high priority at the ongoing LHC and future high-luminosity LHC projects, and it has motivated consideration of dedicated Higgs boson production facilities [3-5].

These investigations focus on the properties of the Higgs boson in the vacuum. However, most of the Higgs bosons in the early Universe existed in a high-temperature and high-density environment [6,7]. An understanding of the role of the Higgs boson in the early Universe would be advanced through the study of the Higgs boson not only in the vacuum but also in an extreme medium. Heavy-ion collisions at the LHC, proposed to study properties of the quark-gluon plasma (QGP), create an extreme environment with high temperature and density [8]. They are well suited at the same time to study the behavior of the Higgs boson in a hot dense environment.

The expansion and cooldown of the QGP at the LHC is predicted to have a typical timescale of about $10 \mathrm{fm} / c$ [9-11]. Although longer than the lifetime of the electroweak

Published by the American Physical Society under the terms of the Creative Commons Attribution 4.0 International license. Further distribution of this work must maintain attribution to the author(s) and the published article's title, journal citation, and DOI. Funded by SCOAP.
(EW) gauge bosons and the top quark, this timescale is shorter than the lifetime of the Higgs boson (which is $\sim 47 \mathrm{fm} / c$ ). The consequences include the following. (i) Particles from Higgs decay, which do not travel in the QGP, will carry information on the Higgs boson. (ii) Because the strong backgrounds are reshaped by the QGP medium while the signal is nearly unchanged, the phenomenology of Higgs boson hadronic decay is different from $p p$ collisions. (iii) A check of the first two consequences serves as a natural probe of the Higgs boson lifetime.

In this Letter, we study the production and decays of the Higgs boson in heavy-ion collisions. We point out the main differences with the proton-proton case. Jets produced from hadronic decays of the Higgs boson are not affected much by the QGP, since the decay happens at a much later stage. Meanwhile, jets produced from hard QCD scattering and decays of EW gauge bosons and the top quark experience energy loss through interaction with the medium [12], known as jet quenching, an established phenomenon in heavy-ion collisions at the Brookhaven RHIC facility and the LHC [13]. These different responses lead to the suppression of the SM backgrounds to hadronic decays of the Higgs boson and also to distinct kinematic configurations of the signal and backgrounds, resulting in an enhanced ratio of the signal over the background when compared to $p p$ collisions. We explore different models of jet quenching to provide quantitative estimates for the case of $Z H$ associated production with Higgs decay $H \rightarrow b \bar{b}$. A different perspective on Higgs boson physics in heavy-ion collisions is proposed in Refs. [14,15].

Higgs boson production.-The cross section for Higgs boson production in collisions of two heavy nuclei with charge $Z$ and atomic number $A$ is 


$$
\begin{aligned}
\sigma(A A & \rightarrow H+X) \\
= & A^{2} c(f) \sum_{a, b} \int d x_{a} d x_{b} \\
& \times f_{a / A}\left(x_{a}, \mu_{F}^{2}\right) f_{b / A}\left(x_{b}, \mu_{F}^{2}\right) \hat{\sigma}(a b \rightarrow H+X) .
\end{aligned}
$$

Here $f_{i / A}\left(x_{i}, \mu_{F}^{2}\right)$ is the effective nuclear parton distribution function (PDF) of parton $i$ carrying momentum fraction $x_{i}$ of the nucleon at a factorization scale $\mu_{F} ; \hat{\sigma}$ is the partonic cross section; and $A^{2} c(f)$ is the number of nucleon collisions for the centrality range $f$, for which $c(f)$ can be obtained by integrating the overlap function of the two nuclei over the corresponding range of impact parameters [16]. For the centrality range $0 \%-10 \%$ in this study, $c(f)$ is calculated to be $42 \%$ with the Glauber Monte Carlo model [16] for PbPb collisions at $\sqrt{s_{N N}}=5.5 \mathrm{TeV}$. In Table I, we show cross sections for Higgs boson production in different channels for $\mathrm{PbPb}$ collisions at the LHC, HE-LHC, and FCC- $h h$ [17] or SPPC [5], with $\sqrt{s_{N N}}=5.5,11$, and $39.4 \mathrm{TeV}$, respectively. We calculate the partonic cross sections with MCFM $[18,19]$ to next-to-leading order in QCD for vector boson fusion (VBF) and next-to-next-toleading order (NNLO) for gluon fusion (GF) and for associated production. The cross sections for production in gluon fusion agree well with those shown in Ref. [14] apart from differences due to scale choices. The centrality factors are similar for the three energies and are not applied in Table I. For comparison, cross sections for production in $p p$ collisions are also listed in Table I.

We focus on decays of the Higgs boson to bottom quarks for which the associated production with a $Z$ boson and its subsequent leptonic decay gives the strongest sensitivity $[20,21]$, albeit with a relatively small cross section. The dominant backgrounds in this case are $Z$ plus bottom-quark pair production and top-quark pair production with leptonic decays. Bottom quarks from decays of the Higgs boson form two energetic jets that can be detected with various $b$-tagging algorithms [22]. On the other hand, in the environment of heavy-ion collision, $b$ jets from the backgrounds will lose energy from interactions in the QGP [13].

TABLE I. Cross sections for Higgs boson production from different processes in $\mathrm{PbPb}$ collisions and proton-proton collisions at $\sqrt{s_{N N}}=5.5,11$, and $39.4 \mathrm{TeV}$, respectively. The nCTEQ15 PDFs [28] and CT14 PDFs [29] are used for the $\mathrm{PbPb}$ and $p p$ collisions, respectively.

\begin{tabular}{cccc}
\hline \hline & \multicolumn{3}{c}{$\mathrm{PbPb}(p p)$ in nb $(\mathrm{pb})$} \\
\cline { 2 - 4 } Process & $5.5 \mathrm{TeV}$ & $11 \mathrm{TeV}$ & $39.4 \mathrm{TeV}$ \\
\hline $\mathrm{GF}$ & $480(10.2)$ & $1556(35.2)$ & $9580(235)$ \\
$\mathrm{VBF}$ & $15.3(0.316)$ & $65.6(1.40)$ & $421(10.02)$ \\
$Z H$ & $10.2(0.230)$ & $28.1(0.687)$ & $147(3.97)$ \\
$W^{+} H$ & $8.38(0.162)$ & $21.8(0.716)$ & $94.2(3.19)$ \\
$W^{-} H$ & $9.22(0.143)$ & $23.4(0.435)$ & $99.5(2.34)$ \\
\hline \hline
\end{tabular}

Owing to the dead-cone effect of QCD radiation [23], it has been argued that a primary $b$ quark will lose less energy than light quarks when traversing QGP, but experimental measurements have shown a similar level of nuclear suppression for inclusive jets and $b$ jets and similar distortion of transverse momentum balance [22,24] of dijets from jet quenching. The fraction of energy lost from a primary $b$-quark jet is thus believed to be comparable to that from a light quark, at least for jets with high transverse momentum. There are also theoretical studies supporting the similarity of quenching of jets initiated by $b$ quarks and light quarks [25-27].

Jet-quenching models.-We base our quantitative estimates on simplified phenomenological models of jet quenching, since a full Monte Carlo generator with jet quenching is not available for the processes of interest. (Such generators exist for QCD jet production, prompt photon production, and electroweak boson plus a single jet production [30-32].) Differences among the three models provide a measure of the uncertainties in our results. The average loss of transverse momentum for a jet traversing the QGP compared to the vacuum is parametrized with a convenient form

$$
\left\langle\delta p_{T}\right\rangle=a p_{T}+b \ln \left(p_{T} / \mathrm{GeV}\right)+c .
$$

The parameters depend on the center of mass energy, the collision centrality, and also the jet reconstruction scheme. In the following, we use the anti- $k_{T}$ [33] algorithm with $R=0.3$. The choice of small jet cone size is typical for a heavy-ion collision in order to minimize effects of fluctuations due to underlying events. We choose three representative models for quark jets in $\mathrm{PbPb}$ collisions with a centrality class of $0 \%-10 \%$, i.e., with strong quenching $a=0, b=2 \mathrm{GeV}, \quad c=12 \mathrm{GeV}$, medium quenching $a=0.15, b=c=0$, and mild quenching $a=b=0$, $c=10 \mathrm{GeV}$. These choices correspond to a loss of transverse momentum of 21,15 , and $10 \mathrm{GeV}$, respectively, for a jet with $p_{T}=100 \mathrm{GeV}$ in a vacuum. The model with medium quenching was used previously in a study of top-quark pair production in heavy-ion collisions [12], except there the scaling was applied on individual constituent particles. In addition, we impose Gaussian smearing on the energy loss to mimic the fluctuations in jet quenching with the width set to half of the average energy lost. The jet energy resolution is parametrized as

$$
\sigma\left(p_{T}\right)=\sqrt{C^{2}+\frac{S^{2}}{p_{T}}+\frac{N^{2}}{p_{T}^{2}}} .
$$

Representative values of the $C, S$, and $N$ parameters from $\mathrm{CMS}$ for different centrality classes in $\mathrm{PbPb}$ collisions can be found in Ref. [34] and are used in our calculations. In $\mathrm{PbPb}$ collisions, subtraction of underlying events is performed and contributes to the $N$ term in the jet energy resolution and also a deterioration of the $S$ term. 


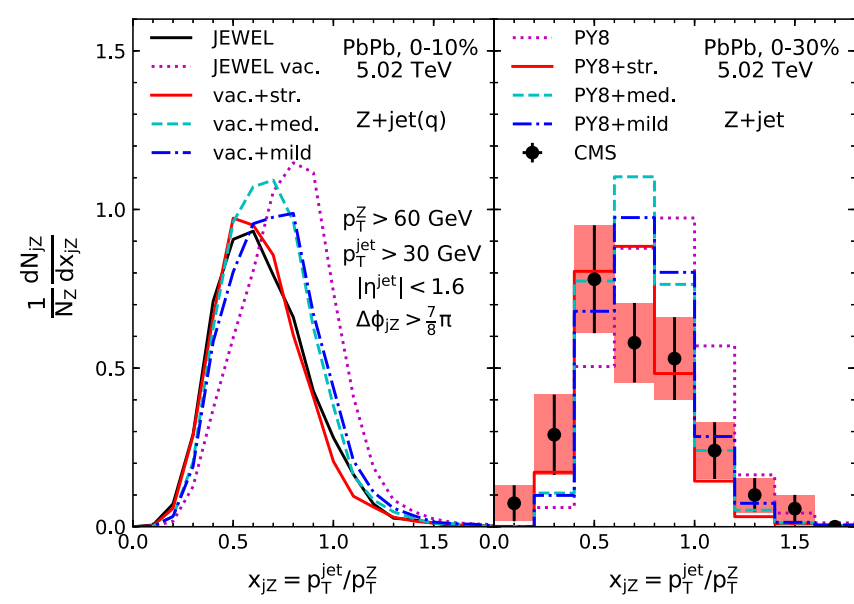

FIG. 1. The impact of different models on jet observables is shown taking, as an example, production of a $Z$ boson plus a single jet. Distribution of the ratio of transverse momenta in $Z+$ jet production in $\mathrm{PbPb}$ collisions. Left: Comparison of predictions from JEWEL2.0 and the folded results with various models, for centrality class $0 \%-10 \%$ and only quark final states included; right: comparison of the folded results with CMS measurement for centrality class $0 \%-30 \%$.

The transverse momentum imbalance in $Z$ boson plus jet production was measured recently by the CMS Collaboration in $\mathrm{PbPb}$ collisions at $\sqrt{s_{N N}}=5.02 \mathrm{TeV}$ as a hard probe of jet quenching [35]. Following the analysis in Ref. [35], we plot in Fig. 1 distributions of the ratio of the transverse momenta $x_{j Z}=p_{T}^{\text {jet }} / p_{T}^{Z}$ normalized to the rate of inclusive $Z$ boson production, where $p_{T}^{\text {jet }}$ is the transverse momentum of the leading jet. In the plot on the left side in Fig. 1, we show predictions from the Monte Carlo program JEWEL 2.0.0 [32] for the centrality class $0 \%-10 \%$. A prediction without jet quenching (vacuum) is also shown, obtained from PYTHIA 6.4 [36] incorporated in JEWEL 2.0.0. We use only the hard matrix elements for quark final states, since we are interested in quenching of jets initiated by quarks. The initial temperature of the QGP is set to $590 \mathrm{MeV}$ [37]. A shift to lower values is seen in the distribution as quenching is increased, as well as a reduction of the event rate. For comparison with the JEWEL prediction, we also show predictions obtained by applying our quenching models to the vacuum calculation on an event-by-event basis. The folded result with strong quenching is in good agreement with the JEWEL result. In the plot on the right in Fig. 1, we compare our folded results with the CMS data measured for centrality class $0 \%-30 \%$ [35]. The baseline vacuum prediction is from PYTHIA 8 [38] with both gluon and quark final states included; the latter contributes more than $80 \%$ of the total production rate. The CMS data disfavor the vacuum prediction. The three simplified quenching models are consistent with current data.

Signal and backgrounds. - We consider the signal process $\mathrm{PbPb} \rightarrow Z H \rightarrow \ell^{+} \ell^{-} b \bar{b}$, in the $0 \%-10 \%$ centrality class, with $\ell=e, \mu$ for which the QCD backgrounds are highly suppressed. We simulate the signal and backgrounds at leading order using SHERPA 2.2.4 [39], including parton showering and hadronization, and with nCTEQ15 PDFs [28]. The dominant SM backgrounds are $Z b \bar{b}$ production and $t \bar{t}$ production with leptonic decays of top quarks. Other $\mathrm{SM}$ backgrounds including those from production of $Z$ plus light flavors are significantly smaller and are ignored. We normalize the total cross sections of the signal to the NNLO values in Table I, and of the $t \bar{t}$ background to the NNLO predictions with resummed corrections from ToP+ +2.0 [40,41], times the relevant centrality factors. The Monte Carlo events are passed to RIVET [42] for analysis with an anti- $k_{T}$ jet algorithm as implemented in FASTJET [43] and a distance parameter of 0.3. Jet quenching and jet energy resolution are applied according to Eqs. (2) and (3). We use preselection cuts similar to those in the CMS heavyion analysis [35]:

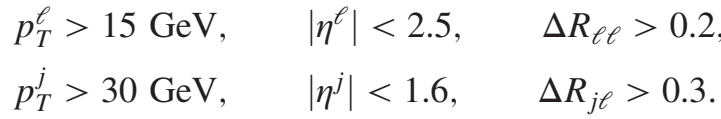

We select events in the following signal-like region: (i) a pair of same-flavor opposite-sign charged leptons with invariant mass $\left|m_{\ell \ell}-m_{Z}\right|<10 \mathrm{GeV}$; (ii) exactly two jets, both $b$-tagged, with separation $\Delta R_{b b}<2.0$; (iii) the transverse momentum of the reconstructed vector boson $p_{T}^{Z} \equiv p_{T}^{\ell \ell}>100 \mathrm{GeV}$. We assume a $b$-tagging efficiency of $80 \%$, better than that achieved in the CMS analysis [22] but expected in future runs. The requirement of large $p_{T}^{Z}$ can suppress the $t \bar{t}$ background efficiently.

The analysis so far follows Ref. [20]. As mentioned earlier, different quenching properties of the signal and backgrounds lead to further separation in certain variables. Separation is illustrated in Fig. 2 for the ratio $x=p_{T}^{b \bar{b}} / p_{T}^{Z}$

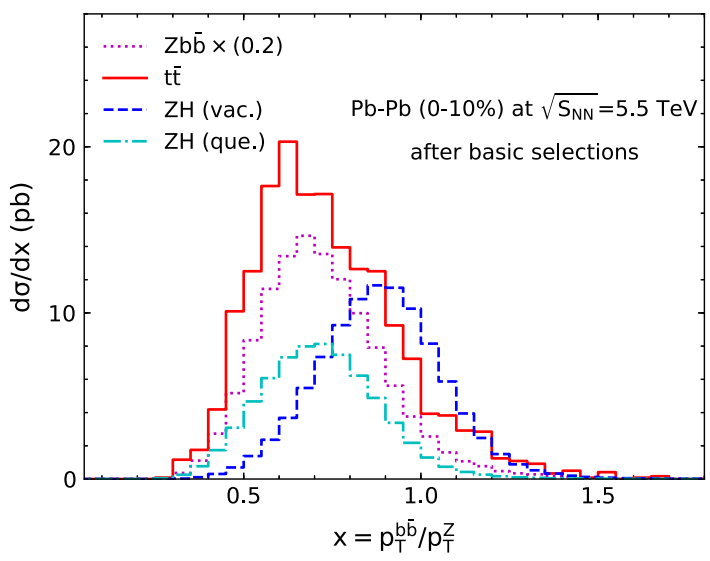

FIG. 2. Distributions of the ratio of the transverse momenta of the pair of $b$ jets and the $Z$ boson for $\mathrm{PbPb}$ collision with $\sqrt{s_{N N}}=$ $5.5 \mathrm{TeV}$ and centrality class $0 \%-10 \%$, after basic selections. For the nominal case, both backgrounds are strongly quenched, while the signal is unquenched. The distribution for a quenched signal is also shown as a comparison. The $Z b \bar{b}$ result has been multiplied by 0.2 . 


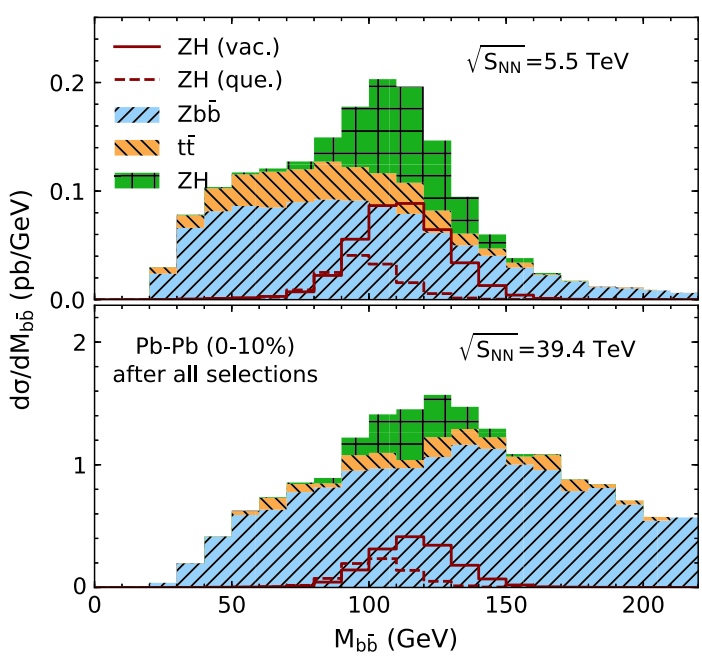

FIG. 3. Distributions of the invariant mass of the pair of $b$ jets after all selections, similar to Fig. 2.

of the transverse momenta of the reconstructed $b \bar{b}$ pair and the $Z$ boson. We apply the strong quenching model on the two backgrounds, and the signal is vacuumlike. The backgrounds tend to peak in the region of smaller $x$, since both of the $b$ jets lose a fraction of their energies. In Fig. 2, we also show the result for the extreme case in which the $b$ jets in the signal process are also strongly quenched. In this case, besides the shift of the peak, the signal normalization is also reduced, since more $b$ jets fall below the $p_{T}$ threshold. Not shown here, we find that the transverse momentum of the (sub)leading jet shows similar separation power.

To establish the discovery potential of the signal, we demand events with $x>0.75$, and $p_{T}>60 \mathrm{GeV}$ for the leading jet at LHC and HE-LHC and for the subleading jet at FCC- $h h$. The invariant-mass distribution of the two $b$ jets $M_{b \bar{b}}$ is shown in Fig. 3 after all selections. The dominant background is $Z b \bar{b}$, and the signal exhibits a clear peak near

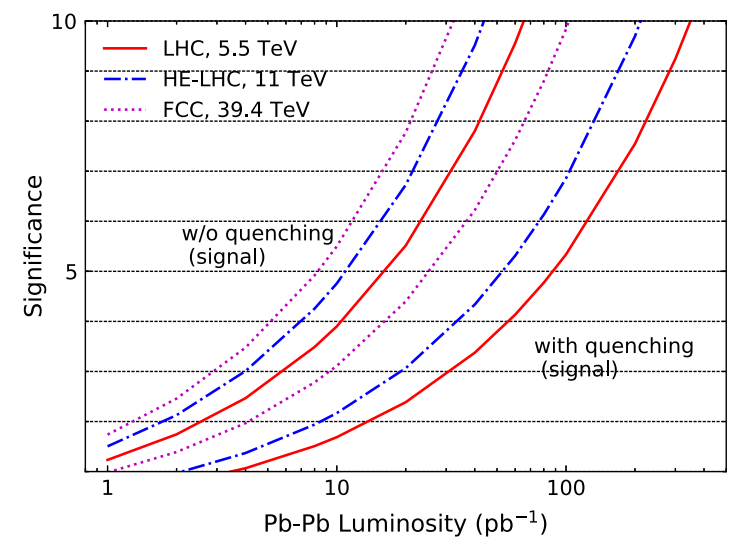

FIG. 4. Expected significance of the Higgs boson signal as a function of the ion luminosity for $\mathrm{PbPb}$ collisions at $\mathrm{LHC}$, HE$\mathrm{LHC}$, and FCC- $h h$. Results for the case of a quenched signal are also shown for comparison.
TABLE II. Ion luminosity required to reach $5 \sigma$ significance for the signal for different models of jet quenching and collision energies. Numbers in parentheses correspond to $3 \sigma$ evidence.

\begin{tabular}{lcccc}
\hline \hline Lumi $\left(\mathrm{pb}^{-1}\right)$ & Strong & Medium & Mild & Vacuum \\
\hline LHC & $16(5.9)$ & $27(9.8)$ & $26(9.3)$ & $48(17)$ \\
HE-LHC & $11(4.0)$ & $20(7.2)$ & $20(7.2)$ & $34(12)$ \\
FCC- $h h$ & $8.0(2.9)$ & $13(4.7)$ & $14(5.0)$ & $22(8.0)$ \\
\hline \hline
\end{tabular}

the Higgs boson mass. The large width of the signal reflects the effects of jet energy smearing. In Fig. 3, we also display the signal distribution for the case of strong quenching. It shows a much weaker peak at a lower mass. A comparison of FCC- $h h$ to LHC shows that the background to signal ratio increases for $Z b \bar{b}$ owing to the higher energy and decreases for $t \bar{t}$ as a result of the cut on the subleading jet.

We use the log-likelihood ratio $q_{0}[44]$ as a test statistic to calculate the expected significance of the signal based on the $M_{b \bar{b}}$ distribution, as a function of the integrated luminosity of the collision program. The results are shown in Fig. 4 and in Table II. For the LHC, a 5(3) $\sigma$ discovery (evidence) requires a total ion luminosity of about 16 (5.9) $\mathrm{pb}^{-1}$ in $\mathrm{PbPb}$ collisions, larger than the projected LHC luminosity [45]. The numbers are $8.0(2.9) \mathrm{pb}^{-1}$ for $\mathrm{PbPb}$ collisions at FCC- $h h$. The significance if the signal is also quenched are much lower than the nominal case shown in Fig. 4. The results for alternative quenching models and for no quenching of the backgrounds are summarized in Table II. The improvement in signal-background discrimination from jet quenching is clear. We expect the sensitivity can be further improved, e.g., by using multivariate analysis and by including $Z$ decays into neutrinos and $W H$ production as demonstrated in Ref. [20]. Taken together, they may bring down the needed luminosity by a factor of 2. Nevertheless, with a much lower luminosity, one can manage to study Higgs boson production in the diphoton channel [14,15], including its interaction with the medium [46].

Summary.-The long lifetime of the Higgs boson relative to the typical timescale of the QGP makes it plausible that the strong decay products of Higgs bosons produced in heavy-ion collisions escape the QGP medium unaffected. On the other hand, QCD backgrounds will be attenuated by jet quenching. These features open the possibility of enhanced ratios of signal to backgrounds. We demonstrated these ideas with the specific example of associated $\mathrm{ZH}$ production in $\mathrm{PbPb}$ collisions at various colliders using simplified models of jet quenching. The integrated luminosities needed for an observation of the signal are $\sim 10 \mathrm{pb}^{-1}$. It will be interesting to investigate the potential of other production channels of the Higgs boson with larger cross sections $[14,15,47]$ and also incorporating information on jet shapes [48-51] expected to be different for quenched and unquenched jets. 
J.G. thanks Lie-Wen Chen for useful discussions. E. L. B.'s work at Argonne is supported in part by the U.S. Department of Energy under Contract No. DE-AC0206CH11357. The work of J. G. and A. J. is supported by the National Natural Science Foundation of China under Contract No. 11875189 and No. 11835005. The work of H. Z. is supported by Institute of High Energy Physics, Chinese Academy of Science, under Contract No. Y6515580U1 and Innovation Grant Contract No. Y4545171Y2.

*berger@anl.gov

†jung49@sjtu.edu.cn

*adil.jueid@sjtu.edu.cn

${ }^{\S}$ zhanghao@ihep.ac.cn

[1] G. Aad et al. (ATLAS Collaboration), Phys. Lett. B 716, 1 (2012).

[2] S. Chatrchyan et al. (CMS Collaboration), Phys. Lett. B 716, 30 (2012).

[3] M. Bicer et al. (TLEP Design Study Working Group), J. High Energy Phys. 01 (2014) 164.

[4] A. Arbey et al., Eur. Phys. J. C 75, 371 (2015).

[5] M. Ahmad et al. (CEPC-SPPC Study Group), Reports No. IHEP-CEPC-DR-2015-01, No. IHEP-TH-2015-01, No. IHEP-EP-2015-01, 2015.

[6] G. Gamow, Phys. Rev. 70, 572 (1946).

[7] R. A. Alpher, H. Bethe, and G. Gamow, Phys. Rev. 73, 803 (1948).

[8] N. Armesto and E. Scomparin, Eur. Phys. J. Plus 131, 52 (2016).

[9] M. Connors, C. Nattrass, R. Reed, and S. Salur, Rev. Mod. Phys. 90, 025005 (2018).

[10] R. Pasechnik and M. Sumbera, Universe 3, 7 (2017).

[11] C. Shen and U. Heinz, Phys. Rev. C 85, 054902 (2012); 86, 049903(E) (2012).

[12] L. Apolinario, J. G. Milhano, G. P. Salam, and C. A. Salgado, Phys. Rev. Lett. 120, 232301 (2018).

[13] G.-Y. Qin and X.-N. Wang, Int. J. Mod. Phys. E 24, 1530014 (2015).

[14] D. d'Enterria, Nucl. Part. Phys. Proc. 289-290, 237 (2017).

[15] D. d'Enterria et al. (FCC-Ions Study Group), Nucl. Phys. A967, 888 (2017).

[16] C. Loizides, J. Kamin, and D. d'Enterria, Phys. Rev. C 97, 054910 (2018).

[17] M. Mangano, CERN Yellow Report No. CERN 2017-003M, 2017.

[18] J. M. Campbell, R. K. Ellis, and C. Williams, J. High Energy Phys. 06 (2016) 179.

[19] R. Boughezal, J. M. Campbell, R. K. Ellis, C. Focke, W. Giele, X. Liu, F. Petriello, and C. Williams, Eur. Phys. J. C 77, 7 (2017).

[20] M. Aaboud et al. (ATLAS Collaboration), J. High Energy Phys. 12 (2017) 024.

[21] A. M. Sirunyan et al. (CMS Collaboration), Phys. Lett. B 780, 501 (2018).

[22] S. Chatrchyan et al. (CMS Collaboration), Phys. Rev. Lett. 113, 132301 (2014); 115, 029903(E) (2015).
[23] D. d'Enterria and B. Betz, Lect. Notes Phys. 785, 285 (2010).

[24] A. M. Sirunyan et al. (CMS Collaboration), J. High Energy Phys. 03 (2018) 181.

[25] J. Huang, Z.-B. Kang, and I. Vitev, Phys. Lett. B 726, 251 (2013).

[26] M. Djordjevic, B. Blagojevic, and L. Zivkovic, Phys. Rev. C 94, 044908 (2016).

[27] F. Senzel, J. Uphoff, Z. Xu, and C. Greiner, Phys. Lett. B 773, 620 (2017).

[28] K. Kovarik, A. Kusina, T. Jeo, D. B. Clark, C. Keppel, F. Lyonnet, J. G. Morfín, F. I. Olness, J. F. Owens, I. Schienbein, and J. Y. Yu, Phys. Rev. D 93, 085037 (2016).

[29] S. Dulat, T.-J. Hou, J. Gao, M. Guzzi, J. Huston, P. Nadolsky, J. Pumplin, C. Schmidt, D. Stump, and C. P. Yuan, Phys. Rev. D 93, 033006 (2016).

[30] M. Arneodo, L. Lamberti, and M. Ryskin, Comput. Phys. Commun. 100, 195 (1997).

[31] I. P. Lokhtin, L. V. Malinina, S. V. Petrushanko, A. M. Snigirev, I. Arsene, and K. Tywoniuk, Comput. Phys. Commun. 180, 779 (2009).

[32] K. C. Zapp, Eur. Phys. J. C 74, 2762 (2014).

[33] M. Cacciari, G. P. Salam, and G. Soyez, J. High Energy Phys. 04 (2008) 063.

[34] A. M. Sirunyan et al. (CMS Collaboration), Phys. Lett. B 785, 14 (2018).

[35] A. M. Sirunyan et al. (CMS Collaboration), Phys. Rev. Lett. 119, 082301 (2017).

[36] T. Sjostrand, S. Mrenna, and P. Z. Skands, J. High Energy Phys. 05 (2006) 026.

[37] R. Kunnawalkam Elayavalli and K. C. Zapp, Eur. Phys. J. C 76, 695 (2016).

[38] T. Sjostrand, S. Ask, J. R. Christiansen, R. Corke, N. Desai, P. Ilten, S. Mrenna, S. Prestel, C. O. Rasmussen, and P.Z. Skands, Comput. Phys. Commun. 191, 159 (2015).

[39] T. Gleisberg, S. Hoeche, F. Krauss, M. Schonherr, S. Schumann, F. Siegert, and J. Winter, J. High Energy Phys. 02 (2009) 007.

[40] M. Czakon and A. Mitov, Comput. Phys. Commun. 185, 2930 (2014).

[41] M. Czakon, P. Fiedler, and A. Mitov, Phys. Rev. Lett. 110, 252004 (2013).

[42] A. Buckley, J. Butterworth, L. Lonnblad, D. Grellscheid, H. Hoeth, J. Monk, H. Schulz, and F. Siegert, Comput. Phys. Commun. 184, 2803 (2013).

[43] M. Cacciari, G. P. Salam, and G. Soyez, Eur. Phys. J. C 72, 1896 (2012).

[44] G. Cowan, K. Cranmer, E. Gross, and O. Vitells, Eur. Phys. J. C 71, 1554 (2011); 73, 2501 (2013).

[45] J. M. Jowett, J. Phys. G 35, 104028 (2008).

[46] D. d'Enterria and C. Loizides (to be published).

[47] E. L. Berger, J. Gao, A. Jueid, and H. Zhang (to be published).

[48] S. Chatrchyan et al. (CMS Collaboration), Phys. Lett. B 730, 243 (2014).

[49] Y.-T. Chien and I. Vitev, J. High Energy Phys. 05 (2016) 023.

[50] L. Apolinario, J. G. Milhano, M. Ploskon, and X. Zhang, Eur. Phys. J. C 78, 529 (2018).

[51] H. T. Li and I. Vitev, arXiv:1801.00008. 